\title{
Nitrogen metabolism in plants under low oxygen stress
}

\author{
Anis M. Limami · Houssein Diab · Jérémy Lothier
}

Received: 6 December 2013 / Accepted: 14 December 2013 / Published online: 27 December 2013

(C) Springer-Verlag Berlin Heidelberg 2013

\begin{abstract}
More frequent flooding and waterlogging events due to more heavy precipitation are expected worldwide in the context of climate change. Accordingly, adaptation of plants to oxygen limitation at both cellular and whole plant levels should be investigated thoroughly, that derived knowledge could be taken into account in breeding programs and agronomical practices for saving plant fitness, growth and development even when oxygen availability is low. In the present review, we highlight current knowledge on essential aspects of low oxygen stress-induced changes in nitrogen metabolism. The involvement of two possible pathways for NO production either via the reaction catalyzed by nitrate reductase or at Complex III or IV of the mitochondrial electron transport chain, thus contributing to ATP synthesis via the so-called nitrite-NO respiration, is discussed. NO is proposed to be scavenged by non-symbiotic hemoglobin $(\mathrm{Hb})$ in a $\mathrm{Hb} / \mathrm{NO}$ cycle, in which $\mathrm{NAD}(\mathrm{P}) \mathrm{H}$ is oxidized for the conversion of $\mathrm{NO}$ into $\mathrm{NO}_{3}{ }^{-}$. The investigation of an additional adaptation to the decrease in oxygen availability via transcriptional and posttranslational regulation of amino acid synthesis pathways, using publicly available transcriptome and translatome data for Arabidopsis thaliana and rice is also discussed.
\end{abstract}

\section{Introduction}

Flooding and waterlogging due to heavy rainfall are expected to become more frequent as a consequence of

A. M. Limami $(\bowtie) \cdot$ H. Diab $\cdot$ J. Lothier

University of Angers, UMR 1345 IRHS, SFR 4207 QUASAV,

2 Bd Lavoisier, 49045 Angers, France

e-mail: anis.limami@univ-angers.fr

A. M. Limami $\cdot$ H. Diab $\cdot$ J. Lothier

INRA, UMR 1345 IRHS, 2 Bd Lavoisier, 49045 Angers, France climatic change (The Intergovernmental panel on climatic change http://www.ipcc.ch/). In this context, plants are threatened by a reduction of the availability of oxygen, during more or less prolonged periods, due to the slower diffusion of oxygen through water and continuing competition for oxygen with respiring microorganisms (Jackson 1985; Drew 1997). Under hypoxic conditions, oxygen concentration in the cells becomes too low to support aerobic respiration, resulting in energy deficit and ultimately cell and tissue death, particularly in plants that are not adapted to flooding and waterlogging (Jackson and Armstrong 1999; Gout et al. 2001; Bailey-Serres and Voesenek 2008; BaileySerres et al. 2012; Licausi 2013). Major disorders caused by low oxygen stress are related to energy crisis caused by the inhibition of mitochondrial oxidative phosphorylation and subsequent reduction in ATP production. Thus, available energy becomes insufficient to meet the needs of energy-demanding biological processes (Greenway and Gibbs 2003). One of the major consequences of the shortage in ATP is an impaired functioning of the plasma membrane $\mathrm{H}^{+}$pumping ATPase that contributes to hypoxiainduced acidification of the cytoplasm (Felle 2006).

Adaptation of the metabolism of plants to low oxygen stress is generally considered in terms of anaerobic carbon metabolism in relation to glycolysis, fermentation and energy homeostasis as illustrated by the numerous articles further cited in the introduction of this review. However, although only alanine aminotransferase (AlaAT) was found among the core hypoxia-response genes in Arabidopsis (Mustroph et al. 2009), nitrogen metabolism was shown to contribute cellular acclimation to low oxygen stress in plants (Bailey-Serres et al. 2012; Bailey-Serres and Voesenek 2008). The present review considers the effect of low oxygen stress on several processes related to $\mathrm{N}$ metabolism, i.e., nitrate absorption, nitrate and nitrite reduction, 
nitric oxide (NO) production, ammonium assimilation and amino acids metabolism and their contribution to low oxygen stress tolerance.

Cross-kingdom comparison of transcriptomic adaptation to low oxygen stress showed that changes associated with glycolysis, fermentation and alternative respiration probably aimed at limiting damaging effects of energy crisis are among the conserved responses shared by 21 organisms from four kingdoms, Plantae, Animalia, Fungi and Bacteria (Mustroph et al. 2010). Survey of 19 translatomes and three transcriptomes specific to various cells populations and organs of Arabidopsis exposed to a brief period of hypoxia identified a group of 49 genes that are prioritized for translation across cell types of both shoots and roots (Mustroph et al. 2009). Several genes among these 49-core hypoxia-responsive genes encode proteins associated with reconfiguration of metabolism to increase substrate level ATP production and fermentation for $\mathrm{NAD}^{+}$regeneration, i.e., sucrose synthase (SUS4), putative pyrophosphate-fructose-6-phosphate 1-phosphotransferase $(P F P)$, pyruvate decarboxylase ( $P D C 1$ and $P D C 2)$, alcohol dehydrogenase $(A D H 1)$ and putative lactate transporter (Mustroph et al. 2009). Concerted activation of both glycolysis and alcoholic fermentation allows for ATP production and NAD ${ }^{+}$ regeneration to maintain glycolysis (Albrecht et al. 2004; Ismond et al. 2003; Kursteiner et al. 2003; Drew 1997). As an energy-saving strategy SUS is used instead of invertase to funnel hexose to glycolysis and PPi-dependent enzymes are preferred to ATP-dependent enzymes, PFP is used instead of phosphofructokinase (PFK) for fructose-1-6-bis$\mathrm{P}$ synthesis and pyruvate phosphate dikinase (PPDK) is used instead of pyruvate kinase (PK) for pyruvate synthesis (Liu et al. 2005b; Narsai et al. 2009).

Unlike animals and microorganisms, direct oxygen sensing has not been found in plants. Instead it is thought that oxygen availability in plants is sensed via changes in redox status (NADH/NAD ratio), reactive oxygen species (ROS), NO, and energy status of the cell (ATP content). It is only recently that the N-end rule pathway (NERP) for protein destabilization described first in animals $(\mathrm{Hu}$ et al. 2005) was proposed as an indirect mechanism for oxygen sensing in plants (Gibbs et al. 2011; Licausi et al. 2011). Transcription factors RAP2.2, RAP2.12, HRE1 and HRE2 of the group-VII Ethylene Response Factors (ERF) revealed as key players in the regulation of low oxygen stress-responsive genes by experiments aiming at comprehensive insight into Arabidopsis transcriptome/translatome responses to hypoxia and anoxia proved to be subjected to oxygen-dependent posttranslational modification through the NERP for protein degradation (Licausi et al. 2010, 2011; Gibbs et al. 2011). Taking into account the fact that $R A P 2.2$ and $R A P 2.12$ are constitutively expressed while HRE 1 and HRE2 are induced by hypoxia, it was proposed that as cellular oxygen concentration decrease, constitutively expressed RAP2.2 and RAP2.12 escape posttranslational modification and $\mathrm{N}$-end rule-mediated proteolysis. They are transported to the nucleus where they switch on hypoxia response by stimulating the expression of secondary ERFs such as HRE1 and HRE2 that stimulate the expression of sets of downstream hypoxia-response genes (Sasidharan and Mustroph 2011; Licausi et al. 2013). Upon re-oxygenation, both constitutively expressed and hypoxiainduced group VII ERFs are destabilized.

$R A P 2.12$ is constitutively expressed throughout the entire plant and further up-regulated by hypoxia but not by the ethylene precursor 1-aminocyclopropane 1-carboxylic acid (ACC) (Licausi et al. 2011). RAP2.2 is also constitutively expressed with high expression in the roots and weak expression in the shoots where it is induced by darkness (Hinz et al. 2010). ACC and ethephon treatments as well as studies on ethylene signal transduction mutants showed that $R A P 2.2$ is induced by ethylene and functions in an ethylene-controlled signal transduction pathway (Hinz et al. 2010). HRE1 and HRE2 were shown to be hypoxia inducible genes (Licausi et al. 2010), however, while HRE2 was insensitive to ethylene, HRE1 was induced by ethylene (Hess et al. 2011) or by ACC (Yang et al. 2011) under aerobic condition and showed hyper-induction when ACC treatment was combined to hypoxia (Yang et al. 2011).

Genes of anaerobic carbon metabolism and energy homeostasis that proved to be important for survival of low oxygen stress were shown to be under the control of groupVII ERFs. Arabidopsis mutants atel/ate2 and prt6 affected in NERP exhibited constitutive expression of more than half of the 49 core hypoxia-induced genes among which several are representatives of anaerobic carbon metabolism $A D H 1, P D C 1, S U S 1, S U S 4$. It is important to note that the expression of these genes under aerobic condition was as high as that in wild type under anaerobic condition (Gibbs et al. 2011). In Arabidopsis seedlings, overexpressing RAP2.2 (Hinz et al. 2010) or its closest homolog RAP2.12 (Licausi et al. 2011) anaerobic carbon metabolism genes were strongly induced during low oxygen stress; changes in gene expression were accompanied by improved survival of the seedlings to flooding-induced low oxygen stress. Microarray analysis followed by quantitative RT-PCR for the comparison of genes induction by hypoxia treatment in wild type and Arabidopsis silenced in both RAP2.2 and $R A P 2.12$ showed a reduced induction of anaerobic carbon metabolism genes in miRNA lines and confirmed that these transcription factors are required for the regulation of hypoxia-induced genes (Licausi et al. 2011; Hinz et al. 2010). Interestingly, the overexpression of $R A P 2.2$ and $R A P 2.12$ did not affect the expression of hypoxia-induced genes when seedlings were grown under aerobic condition indicating that additional mechanisms interfere with the 
signaling pathway controlled by these transcription factors. In fact, it was shown that RAP2.12 and RAP2.2 are submitted to posttranslational modification that leads to their degradation through NERP under aerobic condition (Licausi et al. 2011).

Transcriptome analysis of Arabidopsis lines overexpressing HRE1 and HRE2 in comparison with the wild type under aerobic and hypoxic conditions showed that overexpression of HRE1, but not HRE2, increased the induction of most anaerobic genes under hypoxia (Licausi et al. 2010). By quantitative PCR analysis, the authors showed that overexpression of HREI enhanced the effect of hypoxia on the expression of a significant subset of anaerobic carbon metabolism genes, e.g., ADH1, SUS1 and SUS4. Only the expression of $A D H 1$ was higher in the HRE1 overexpressing line than that in wild type, under aerobic conditions. The role of HRE1 in low oxygen stress was confirmed in Arabidopsis RNAi knockdown mutants (hre 1), which showed reduced hypoxic induction of glycolytic and fermentative genes (Yang et al. 2011). However, in the study of Licausi et al. (2010) null mutants affected in either HRE1 (hrel) or HRE2 (hre2) were not impaired in the expression of hypoxia marker genes while the double mutant (hrel/hre2) showed a drastic decrease in the expression of maker genes after the onset of hypoxia. It was therefore proposed that HRE1 and HRE2 play a partially redundant role in low oxygen signaling in Arabidopsis, improving the tolerance of the plant to the stress by enhancing anaerobic genes expression and ethanol fermentation (Hess et al. 2011; Licausi et al. 2010).

Transcription factors belonging to the sub-group VII of ERF family were shown to control strategies to survive flooding in rice. Two contrasted strategies: the 'quiescence' and the 'escape' strategy were very well dissected in lowland and deepwater rice lines. The first strategy observed also in species like Rumex palustris naturally adapted to face rapid and complete submergence for few weeks, is triggered by an ERF of the sub-group VII, SUB1A (submergence tolerance) (Xu et al. 2006). Only few cultivars highly tolerant and able to survive up to 2 weeks of complete submergence express SUB1A. SUB1A promotes the accumulation of two negative regulators of GA responses SLENDER RICE 1 (SLR1) and SLENDER RICE LIKE 1 (SLRL1), heightens sensitivity to ABA and inhibits growth by inhibiting cell elongation thus decreasing metabolism and energy consumption. Another escape strategy observed in species like Rumex acetosa naturally adapted to progressive and long-term partial submergence consists in a SUB1A-independent enhanced stem internode or petiole elongation to allow at least part of the shoot to be above the surface of water. Shoot elongation under submergence condition is triggered by ethylene signaling pathway and two ERFs of the sub-group VII, SNORKEL1 and SNORKEL2
(SK1 and SK2) (Hattori et al. 2009). The increase in the active form of gibberellin (GA1) in the internodes following submergence suggested GA biosynthesis as a target of SK signaling in the process of internode elongation. Sugar degradation, glycolysis and fermentation were increased in consequence to enable germination and growth under low oxygen condition (Hattori et al. 2009). In coleoptiles of rice growing under low oxygen the abundance of SUS4, enzymes involved in several steps of glycolysis, e.g., pyrophosphate (PPi)-Fru-6-P 1-phosphotransferase, Fructose-bisP aldolase, triosephosphate isomerase, glyceraldehyde-3-phosphate dehydrogenase and enzymes of ethanolic fermentation, ADH1 and PDC1 increased significantly compared to aerobic condition (Shingaki-Wells et al. 2011). Up-regulation of hypoxia-induced genes of sugar metabolism was mediated by the protein kinase CIPK15 (Calcineurin B-like protein kinase interfering protein kinase) that activated the stress sensor SnRK1A (sugar non-fermenting related protein kinase) thus connecting sensing of $\mathrm{O}_{2}$-deficiency signal to the flooding response of rice seedling (Lee et al. 2009).

\section{Nitrate uptake and reduction}

Several studies have shown that nitrate fertilization improves tolerance to hypoxia and more generally limits the effect of a stress caused by a lack of oxygen in the roots (Allègre et al. 2004; Morard et al. 2004; Horchani et al. 2010). There is a paucity of data on nitrate uptake under oxygen-limiting conditions and published works on this topic are often contradictory. It was shown that in wheat $\mathrm{N}$ uptake from nutrient solutions containing either nitrate alone or nitrate plus ammonium decreases when there is a lack of oxygen in the rhizosphere (Buwalda and Greeway 1989). Nitrate uptake by soybean was found to be lower under hypoxic conditions than under aerobic medium (Oliveira and Sodek 2013; Oliveira et al. 2013). In contrast, several studies showed that nitrate uptake by tomato plants increased under prolonged periods of root hypoxia (Morard et al. 2004; Horchani et al. 2010).

The beneficial effect of nitrate supply is probably exerted through the action of nitrate reductase (NR), a cytosolic enzyme that catalyzes NADH-dependent nitrate reduction into nitrite. Nitrate reduction may contribute to cellular acclimation to low oxygen deprivation by regenerating $\mathrm{NAD}^{+}$from NADH. Accordingly, species tolerant to oxygen deprivation exhibit higher NR activity than sensitive ones (Bailey-Serres and Voesenek 2008). Hypoxia-induced acidification of cytosolic pH may increase NR activity by virtue of its low pH optimum (Botrel and Kaiser 1997; Stoimenova et al. 2007). Both in tobacco and in tomato it has been shown that the absence or the decrease in NR activity 
in genetically modified plants or following the addition of tungstate (a potent inhibitor of NR activity) enhances the symptoms of hypoxia. These symptoms are characterized by an increase in the production of both ethanol and lactate accompanied by an accumulation of soluble sugar, an acidification of the cytosol, by plant epinasty chlorosis and a reduction in plant growth (Horchani et al. 2010; Stoimenova et al. 2003). Conversely, it was observed that maize seedlings supplied with nitrate during anoxia maintained a slightly higher cytosolic pH (Libourel et al. 2006).

The increase in NR activity in hypoxic roots is triggered by both transcriptional and posttranslational regulations. NR gene expression was shown to increase in response to root hypoxia in several plant species, e.g., Arabidopsis (Loreti et al. 2005), rice (Lasanthi-Kudahettige et al. 2007), tobacco (Botrel and Kaiser 1997; Stoimenova et al. 2007), and tomato (Allègre et al. 2004). In Cucumis sativus, hypoxic stress caused several-fold increases in the expression of $P P 2 A$, a gene encoding the phospho-NR phosphatase responsible of the activation of NR by dephosphorylation (Shi et al. 2008). Accordingly, NR was highly phosphorylated in aerated roots of hydroponically grown barley and largely inactive, whereas it was partly dephosphorylated and active in hypoxic or anoxic roots. Botrel and Kaiser (1997) observed that rapid anaerobic activation of the NR enzyme following the modification of its phosphorylation state was not triggered by changes in the pool of ATP and AMP, but rather triggered by altered cytosolic $\mathrm{pH}$.

\section{Nitrite reduction and nitric oxide production}

Nitrite content increases in hypoxic tissue and significant amounts of nitrite were shown to be released in the external medium by hypoxic roots or transported through xylem sap to the aerated shoots (Allègre et al. 2004; Morard et al. 2004; Shi et al. 2008; Horchani et al. 2010). The accumulation of nitrite under root hypoxia is the result of the inverse relationship between NR and nitrite reductase (NiR) activities: while NR is stimulated, in some cases NiR is partly inhibited slowing down the rate of nitrite reduction (Shi et al. 2008; Oliveira and Sodek 2013). However, nitrite is not only a byproduct of hypoxic $\mathrm{N}$ metabolism in the root, since it is thought that nitrite rather than nitrate per se is responsible of the beneficial effects of nitrate. Nitrite concentration in micromolar range was as effective as nitrate on the adjustment of cytosolic $\mathrm{pH}$ in maize roots exposed to anoxic conditions (Libourel et al. 2006). In Medicago truncatula RNAi transgenic lines impaired for NRI and $N R 2$ gene expression or in wild-type plants treated with tungstate, the reduction of hypoxia-induced NO production in the nodules was partly reversed by the addition of nitrite. This finding strengthens the hypothesis that nitrite rather than nitrate had a positive effect in hypoxic tissue (Horchani et al. 2011). Since nitrite was effective at very low concentration, its role was suggested not to be restricted to the regeneration of $\mathrm{NAD}(\mathrm{P})^{+}$, but rather linked to a regulatory mechanism related to NO emission (Talwar et al. 2012; Ma et al. 2012; Zhang et al. 2007; Libourel et al. 2006).

If it is well established that nitrite in hypoxic tissue is preferentially used for NO synthesis rather than being channeled through the ammonium assimilatory pathway (Morard et al. 2004; Oliveira and Sodek 2013). The metabolic pathway from which NO is produced from nitrite is still matter of debate. NO could be either produced in a two-step mechanism involving successively NR and electron transport chain (ETC) or only during the reaction catalyzed by NR. In M. truncatula nodules fed with nitrite, both pharmacological and genetic approaches showed that NO production was maintained in the absence of NR activity. This finding indicates that NO production is independent of NR activity and that the role of the NR enzyme is only to reduce nitrate. In the same set of experiments, the inhibition of mitochondrial ETC with rotenone, antimycin $\mathrm{A}$, and myxothiazol blocked NO production both under normoxic and hypoxic conditions thus indicating that mitochondrial ETC was directly involved in the reduction of nitrite to NO (Horchani et al. 2011). Based on experiments performed on isolated plant mitochondria, it was proposed that nitrite is the acceptor of electrons generated by the oxidation of $\mathrm{NAD}(\mathrm{P}) \mathrm{H}$ by the $\mathrm{Ca}_{2}{ }^{+}$-sensitive $\mathrm{NAD}(\mathrm{P}) \mathrm{H}$ dehydrogenases located at the inner mitochondrial membrane surface involving Complex III (ubiquinone:cytochrome $c$ reductase) or COX (complex IV) of the ETC. Thus, when NO is produced it contributes to ATP synthesis as the result of proton pumping at the sites of complex III or COX (Stoimenova et al. 2007; Planchet et al. 2005; Gupta and Igamberdiev 2011; Igamberdiev and Hill 2009; Igamberdiev et al. 2010; Benamar et al. 2008; Jacoby et al. 2012). This process called nitrite-NO respiration (NNR) necessitates the transport of nitrite from the cytosol into the mitochondrial matrix through a presently unknown transporter (Horchani et al. 2011) (Fig. 1).

An alternative hypothesis is that nitrite may participate in NO emission by being reduced in the cytosol by NR (Sakihama et al. 2002; Yamasaki et al. 1999; Kaiser et al. 2002). In shoots, it is unlikely that NR participates to NO production due to the high $\mathrm{Km}(100 \mathrm{mM})$ of the enzyme for nitrite and because NO emission is competitively inhibited by nitrate (Rockel et al. 2002). However, NO emission could be induced in leaves exposed to hypoxia/anoxia in the dark or in detached leaves fed nitrite through the petiole and maintained in the dark (Kaiser et al. 2002). Accordingly, the inactivation of NR by maintaining the enzyme in its phosphorylated state by the inhibitors of PP2A decreased the rate of NO emission (Kaiser et al. 2002). 


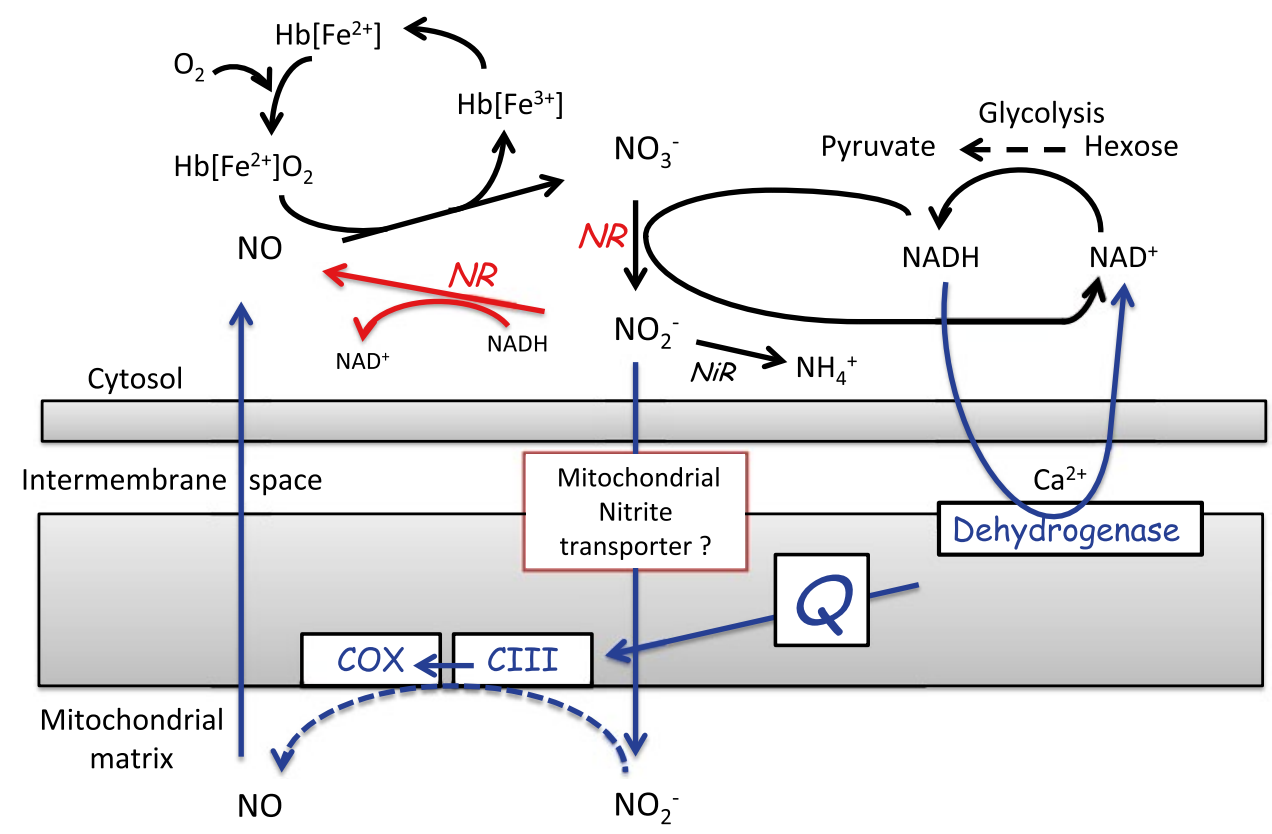

Fig. 1 Simplified schematic representation of two possible pathways for NO production in hypoxic cells. Nitric oxide (NO) is either produced in the cytosol by a side reaction of nitrate reductase when $\mathrm{NiR}$ activity is lower under hypoxic condition and $\mathrm{NO}_{2}^{-}$accumulates (pathway shown in red) or NO is produced in the mitochondrial matrix at levels of oxygen below saturation of cytochrome $c$ oxidase (COX). $\mathrm{NO}_{2}{ }^{-}$transported to the mitochondrial matrix through an unknown transport system serves as an alternative electron acceptor at the sites of complex III and COX. Electrons are generated by

The production of NO in plants could be a side reaction leading to a byproduct of $\mathrm{N}$ metabolism or a physiologically relevant reaction. After several years of investigation, it is believed that NO is perceived in plants as a ubiquitous signal involved in diverse physiological processes including adaptive response to abiotic stresses (BessonBard et al. 2008a, b). For example, in A. thaliana double knock out nial-nia2 NR-deficient mutants were unable to produce NO when submitted to drought stress. In this mutant, the ABA-mediated NO production was dependent on ABA-induced $\mathrm{H}_{2} \mathrm{O}_{2}$ leading to a strong inter-relationship between ABA signaling, endogenous $\mathrm{H}_{2} \mathrm{O}_{2}$ production and NO-induced stomatal closure (Bright et al. 2006). In Nicotiana plumbaginifolia cell suspensions, NO donor promoted an increase in cytosolic free $\mathrm{Ca}^{2+}$ concentration, which was sensitive to intracellular $\mathrm{Ca}^{2+}$ channel inhibitors (Lamotte et al. 2004). It was also shown that in Vicia faba guard cells, $\mathrm{NO}$ selectively regulates $\mathrm{Ca}^{2+}$-sensitive ion channels by promoting $\mathrm{Ca}^{2+}$ release from cellular pools, thus raising cytosolic free cellular $\mathrm{Ca}^{2+}$ content (GarciaMata et al. 2003). These few examples provide strong lines of evidence that hypoxia-induced NO could be involved in a signaling cascade that controls the adaptive response of plants to low oxygen availability. However, more research the action of $\mathrm{Ca}_{2}{ }^{+}$-dependent mitochondrial NADH dehydrogenase that oxidizes NADH produced in the cytosol by glycolytic fermentation and transferred to complex III through ubiquinone (Q) (pathway shown in blue). In both cases, $\mathrm{NO}$ is assumed to be scavenged by oxyhemoglobin $\left[\mathrm{Hb}\left(\mathrm{Fe}_{2}{ }^{+}\right) \mathrm{O}_{2}\right]$ through the $\mathrm{Hb} / \mathrm{NO}$ cycle to regenerate $\mathrm{NO}_{3}{ }^{-}$and metHb $\left[\mathrm{Hb}\left(\mathrm{Fe}_{3}{ }^{+}\right)\right]$. Oxyhemoglobin is regenerated by the spontaneous oxygenation of $\left[\mathrm{Hb}\left(\mathrm{Fe}_{2}^{+}\right)\right]$due to its high affinity for oxygen. Scheme drawn by integrating data from Horchani et al. (2011), Igamberdiev et al. (2004, 2010) and Stoimenova et al. (2007)

is required to identify the various components of this NOmediated signaling pathway and its interplay with NERP (Hu et al. 2005).

The other means by which NO could intervene in the response of plants to hypoxia is the capacity of this molecule to interact with metal-containing proteins (Igamberdiev and Hill 2009). The interaction of NO with hemoglobin $(\mathrm{Hb})$ is not restricted to symbiotic LegHb. Genes encoding non-symbiotic $\mathrm{Hb}$ (class- $1 \mathrm{Hb}$ ) were induced by low oxygen in flooded roots (Mustroph et al. 2009) and NO emission rates were much slower than NO production (Planchet et al. 2005; Stoimenova et al. 2007). It was, therefore, proposed that $\mathrm{Hb}$ might scavenge $\mathrm{NO}$ in hypoxic tissues by catalyzing its turnover to nitrate, thus providing protection against deleterious nitrosative stress (Dordas et al. 2003a, b; Igamberdiev et al. 2004; Igamberdiev and Hill 2004) (Fig. 1). NO was shown to interact with $\mathrm{Hb}$ from different plant species in a $\mathrm{Hb} / \mathrm{NO}$ cycle, in which the excess $\mathrm{NAD}(\mathrm{P}) \mathrm{H}$ is oxidized for the conversion of NO into nitrate (Hebelstrup et al. 2012; Besson-Bard et al. 2008b). When alfalfa plants overexpressing $\mathrm{Hb}$ were compared to wild type or to $\mathrm{Hb}$-antisense plants, symptoms of hypoxia were improved, leading to a longer survival of the Hb overexpressors (Dordas et al. 2003a). NO emission 
was 2.5-fold higher in $\mathrm{Hb}$-silenced plants compared to $\mathrm{Hb}$ overexpressors, suggesting that in plants, $\mathrm{Hb}$ is involved in the response to hypoxia through the modulation of $\mathrm{NO}$ emission and the loss of $\mathrm{N}$ to the atmosphere (Dordas et al. 2003a). Thus, an additional positive effect of $\mathrm{Hb} / \mathrm{NO}$ cycle could be to limit $\mathrm{N}$ loss under $\mathrm{NO}$ emission when oxygen concentration in the medium is close to anoxia (below $0.1 \% \mathrm{O}_{2}$ ) (Hebelstrup et al. 2012).

\section{Amino acid metabolism}

Amino acid metabolism is affected by oxygen and energy shortage, however, the role of accumulated amino acids under low oxygen stress is still elusive (Bailey-Serres and Voesenek 2008; Bailey-Serres et al. 2012). An interesting comparison of protein and metabolite profiling in response to low oxygen stress between rice and wheat coleoptiles showed in rice coleoptiles, but not in wheat coleoptiles increased abundance of a set of enzymes involved in alanine, serine and glycine biosynthesis with the accumulation of these amino acids in rice coleoptiles (Shingaki-Wells et al. 2011). To test whether the accumulation of these amino acids is related to the tolerance of oxygen deprivation in rice, the authors supplemented the medium used for rice and wheat growth with a combination of these amino acids. The results showed a positive effect of exogenous addition of a mix of three (Ser/Ala/Gly) or two (Ser/Ala) amino acids on wheat root tolerance of oxygen deprivation thus indicating that their synthesis could be linked to rice anoxia/hypoxia tolerance (Shingaki-Wells et al. 2011). Metabolic and transcriptomic responses to oxygen availability in rice embryos and young seedlings are in agreement with these findings showing that the changes in enzymes and amino acid contents are sustained by changes in expressions of corresponding genes (Narsai et al. 2009).

To get better insight into amino acid metabolism under low oxygen stress, we used publicly available A. thaliana microarray data related to oxygen deprivation. The data were obtained by several research groups in a variety of experimental setups differing in key determinants of oxygen stress, i.e., $\mathrm{O}_{2}$ contents varying from 0 to $8 \%$, light conditions corresponding to continuous light, darkness or long as well as short day photoperiod. Conditions of application of oxygen stress included seedlings soaked in liquid nutritive medium bubbled with gas enriched in $\mathrm{N}_{2}$ or plantlets growing on solid nutritive medium in sealed tanks gassed with defined gas compositions. All the nutritive media were supplemented with sucrose, the concentration of sugar varied however from 0.5 to $3 \%$. Importantly, the RNA for microarray analyses was extracted from either whole seedlings roots or shoots. Although the experimental conditions were very diverse, AlaAT (At1g17290) expression was ubiquitously stimulated under low oxygen stress. AlaAT was induced under various oxygen stresses and that is probably why AlaAT was the only nitrogen metabolism gene described among the 49-core hypoxiaresponsive genes expressed in various tissues of 7-day old Arabidopsis plantlets grown on solid medium and gassed for $2 \mathrm{~h}$ by argon (99.99\%) (Mustroph et al. 2009). AlaAT expression was similarly induced in Agrobacterium rhizogenes-induced hairy roots soaked in hypoxic $\left(0.5 \% \mathrm{O}_{2}\right)$ liquid medium (Klok et al. 2002), in young seedlings soaked in hypoxic $\left(3 \% \mathrm{O}_{2}\right)$ (Liu et al. 2005a) or anoxic $\left(90 \% \mathrm{~N}_{2}-\right.$ $10 \% \mathrm{H}_{2}$ ) (Loreti et al. 2005) liquid medium and in plantlets grown on solid medium under anoxic (99.99\% argon), or hypoxic ( 1 or $1.5 \% \mathrm{O}_{2}$ ) atmosphere (van Dongen et al. 2009; Hinz et al. 2010). Other nitrogen metabolism genes, without being ubiquitously overexpressed under low oxygen stress, were more or less up-regulated in specific studies, e.g., glutamate dehydrogenase (GDH2; At5g07440), glutamate decarboxylase (GDCl; At5g17330) and glutamate synthase (NADH-GOGAT; At5g53460). These were shown to be induced at early stages of stress $(0.5-6 \mathrm{~h})$ (Klok et al. 2002; Loreti et al. 2005) and inhibited when the stress lasted more than $20 \mathrm{~h}$ (Klok et al. 2002; Yang et al. 2011). Aspartate aminotransferase (ASP2; At5g19550) was also among the induced genes in some studies (Loreti et al. 2005; Van Dongen et al. 2009; Yang et al. 2011).

As transcription factors of group VII ERFs and NERP for protein degradation were shown to be involved in the induction of anaerobic genes (see "Introduction"), it was tempting to browse public transcriptomic data of Arabidopsis mutants to gain further insight on the control of the expression of AlaAT under low oxygen stress. Comparison of the expression of the 49-core hypoxia-responsive genes in seedlings of wild-type Columbia and NERP mutants, ate 1/ate 2 and prt6, under air or brief hypoxia $(2 \mathrm{~h})$ revealed that unlike hypoxia marker genes, e.g., $A D H 1$ (At1g77120), SUS4 (At3g43190) and PDCl (At4g33070), AlaAT (At1g17290 and At1g72330) were not up-regulated under non-stress conditions and did not show stronger expression than in the wild type under low oxygen stress (Gibbs et al. 2011). This result suggests that the regulation of AlaAT by low oxygen stress would be independent of NERP for oxygen sensing in plants. Unlike several hypoxic-responsive genes, AlaAT did not show stronger induction under flooding condition in Arabidopsis lines overexpressing RAP2.12 (Licausi et al. 2011). Transcriptome analyses of Arabidopsis either overexpressing HRE1 and HRE2 or silenced in both genes (hrel/hre2) suggested that induction of AlaAT by hypoxia is not mediated by signaling pathways controlled by these transcription factors (Licausi et al. 2010). However, the expression of AlaAT (At1g17290) was affected in RAP2.2-overexpressing (ox3) and Knockout (rap2.2-2) mutants (Hinz et al. 2010). AlaAT showed 
the same profile of expression in the mutants as hypoxia marker genes ADH1 (At1g77120) and PDCl (At4g33070); it was strongly overexpressed in the shoots of $o x 3$ only under hypoxic-dark treatment but not under hypoxic-light or normoxic-dark treatments (see supplemental Figure 3 in Hinz et al. 2010). It was interesting to observe in the same experiment that $R A P 2.3$ that also belongs to group VII ERFs was induced in a similar manner as AlaAT suggesting its involvement downstream RAP2.2. In total, these results indicate that a hypoxia-induced ethylene-dependent pathway that involves RAP2.2 (with or without RAP2.3) cannot alone induce AlaAT and that other signals related to darkness are also essential. The question we have asked is what might be this or these other signals. The duration of the hypoxic-dark treatment, $5 \mathrm{~h}$ is too short in our opinion to have induced sugar starvation syndrome particularly in the present case where the medium of culture contained $1.5 \%$ sucrose. Two to three days of complete darkness were necessary to induce a sugar starvation state and the expression of the sugar-sensitive gene $G D H 1$ in dark-adapted Arabidopsis (Melo-Oliveira et al. 1996). However, other signals related to the prolonged interruption of photosynthesis, for example disruption of energy homeostasis perceived via ATP/AMP ratio can reasonably be hypothesized. In Arabidopsis, markers of dark-induced (DIN) genes, DINI (At4g35770) and DIN6 (At3g47340) were also induced by flooding/submergence stress in isolated mesophyll cells and whole plants. Fusion of putative promoter of Arabidopsis DIN6 to the luciferase (LUC) gene showed in transfected protoplasts that DIN6-LUC was activated by darkness and hypoxia/submergence within 3-6 $\mathrm{h}$ in a similar way to the endogenous DIN6 gene. Activation was abolished by the protein kinase inhibitor K252a suggesting the requirement of protein kinases in the response to these stress. Cellbased functional screens revealed the specific activation of DIN6-LUC by two ubiquitously expressed members of the SnRK1 (Snf1-related protein kinase 1) group, KIN10 (At3g01090) and KIN11 (At3g29160) but not by two other representatives of this group SnRK2 and SnRK3, despite their established protein kinase activities in osmotic and salt stress responses (Baena-González et al. 2007). Gene expression changes induced by $6 \mathrm{~h}$ of transient KIN10 expression or hypoxia treatment in Arabidopsis protoplasts showed similar profiles. However, expression of AlaAT that was induced in dark and hypoxia-treated protoplasts was only slightly changed in KIN1O overexpressing protoplasts under normoxia. This supports the idea that AlaAT is under the control of convergent signaling pathways (Baena-González et al. 2007). In Fig. 2 we put forward the hypothesis of a cross-talk between hypoxia and darkness signaling pathways for the induction of AlaAT expression. This hypothesis is consistent with the observation that in flooded rice, CIPK15 [calcineurin B-like (CBL)-interacting

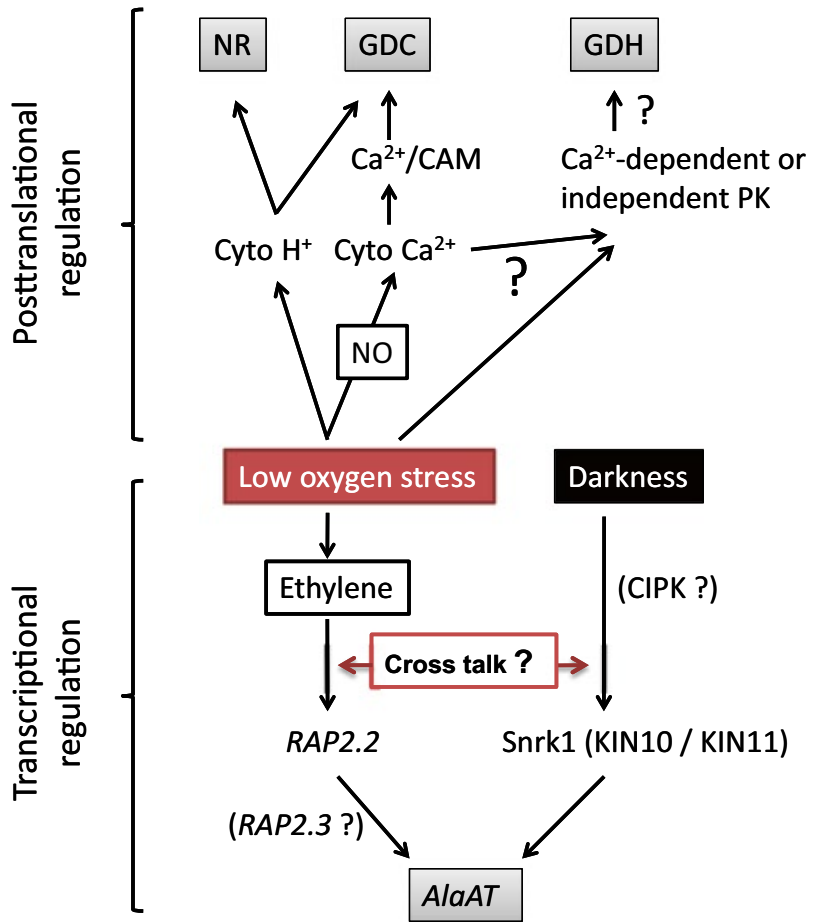

Fig. 2 Summary of several pathways of regulation of nitrogen metabolism by low oxygen stress. Proposition of a hypothetical cross-talk between hypoxia- and darkness-induced signaling pathways for transcriptional regulation of AlaAT. Hypoxia-induced ethylene-dependent pathway involving the transcription factor RAP2.2 was shown to be effective in the induction of AlaAT expression in Arabidopsis shoots only when oxygen stress was associated with darkness (Hinz et al. 2010). Involvement of RAP2.3 downstream of RAP2.2 is questioned since its encoding gene showed the same pattern of regulation as AlaAT under hypoxia-dark treatment in $o x 3$ (RAP2.2 over expressing line) (Hinz et al. 2010). KIN10/KIN11 two members of the Snrk family were shown to mediate darkness and oxygen stress signal in Arabidopsis isolated mesophyll cells and whole plants (Baena-González et al. 2007). CIPK15 was shown to act through Snrk1A for the regulation of sugar fermentation in anoxic rice (Lee et al. 2009). Posttranslational regulation of GDC is triggered by hypoxia-induced increase in cytoplasmic (cyto) $\mathrm{H}^{+}$and $\mathrm{Ca}^{2+}$ concentrations. Hypoxia-induced NO promotes $\mathrm{Ca}^{2+}$ release from cellular pools thus raising free cellular $\mathrm{Ca}^{2+}$ concentration (Garcia-Mata et al. 2003). GDC is activated by acidic $\mathrm{pH}$ and relieved from auto-inhibition by $\mathrm{Ca}^{2+} / \mathrm{CAM}$ complex (Bouché et al. 2004, 2005). NR activity is increased by virtue of its low pH optimum (Stoimenova et al. 2007). Phosphorylation of GDH for decreasing its activity under hypoxic condition is hypothesized based on the posttranslational regulation of this enzyme in crayfish Orconectes virilis (Dawson and Storey 2012)

protein kinase] controls the expression of $A D H$ as a response to low oxygen stress through SnRK1A (Lee et al. 2009).

Although only changes in the expression of AlaAT emerged from transcriptomic studies as part of a consistent and ubiquitous adaptation of primary metabolism to oxygen stress, metabolomic studies on several species showed that the effect of low oxygen stress on amino acid metabolism 
goes beyond alanine accumulation (Bailey-Serres and Voesenek 2008; Bailey-Serres et al. 2012). It has been suggested that the adaptive response of amino acid metabolism to hypoxic stress consists of a concerted modulation of the flux of organic $\mathrm{N}$ going through glutamate and alanine (Limami et al. 2008). Consequently, ATP-consuming pathways such as those involved in glutamine and asparagine biosynthesis are down-regulated in favor of alanine and GABA that generally accumulate, representing the major pool of organic N (Ricoult et al. 2005; Rocha et al. 2010; Bailey-Serres and Voesenek 2008; Narsai et al. 2009). Therefore, it is very likely that modulation of amino acid pathways is the result of posttranslational regulation. It has been shown that GDC activity is responsive to variations in cytoplasmic $\mathrm{H}^{+}$and $\mathrm{Ca}^{2+}$ concentrations that both increase under hypoxia (Fig. 2). GDC activity increases as the cytoplasmic $\mathrm{pH}$ declines and decreases when the $\mathrm{pH}$ again reaches its normal level (Carroll et al. 1994). Furthermore, GDC is relieved from auto-inhibition by the $\mathrm{Ca}^{2+} /$ Calmodulin complex (Bouché et al. 2004, 2005). Decarboxylation of glutamate by GDC is part of the GABA shunt that involves the reactions catalyzed by the enzymes GABA-Transaminase (GABA-T) and succinic semialdehyde dehydrogenase (SSADH). The GABA shunt contributes to cellular $\mathrm{pH}$-stat since glutamate decarboxylation is a proton consuming reaction that increases the $\mathrm{pH}$ (Drew 1997; Greenway and Gibbs 2003). In the freshwater crayfish Orconectes virilis, GDH was shown to be phosphorylated under oxygen-limiting conditions leading to a reduction of the enzyme activity to cut the bridge between $\mathrm{C}$ and $\mathrm{N}$ assimilatory pathways until the fish return to normoxia (Dawson and Storey 2012). If such a posttranslational regulatory mechanism could be effective in plants (Fig. 2), it would explain the discrepancy between the increase in $G D H 1$ gene expression and the decrease in in vivo enzyme activity in $M$. truncatula seedlings under hypoxia (Limami et al. 2008). Induction of GDH1 was interpreted as anticipation on the return to aerobic conditions during which the enzyme could regenerate 2-oxoglutarate by deaminating glutamate during the poststress recovery period (Limami et al. 2008). It has been suggested that a return to aerobic conditions can be anticipated in plants subjected to hypoxic stress by expressing genes for which their translational products have important biological functions during the subsequent period of recovery (Drew 1997). AlaAT activity was measured in vivo by feeding $M$. truncatula seedlings with either ${ }^{15} \mathrm{~N}$-glutamate or ${ }^{15} \mathrm{~N}$-alanine. Under aerobic condition AlaAT catalyzed a reversible reaction, however, under hypoxic condition AlaAT activity was exclusively directed towards alanine biosynthesis using glutamate as amino-N donor and the reverse reaction of glutamate synthesis, using alanine as amino- $\mathrm{N}$ donor, was inhibited thus suggesting a posttranslational regulation (Ricoult et al. 2006).

\section{Conclusion and perspectives}

The present review aimed at showing how nitrogen metabolism intervenes in the ability of plants to mitigate detrimental effects of low oxygen stress at cellular level. It was deliberately focused on domains where research is currently in progress like NO production and amino acid metabolism. In these two areas there are interesting questions waiting for further investigation.

As to nitrate/nitrite reduction and NO production, it seems crucial to determine the mode of transport of nitrite into the mitochondria for better understanding the contribution of nitrite-NO respiration to the amelioration of energy crisis and unraveling the controversy on respective roles of mitochondrial ETC and cytosolic NR in the production of NO.

Concerning the modulation of amino acid synthesis as an adaptation to the decrease in oxygen availability, a thorough investigation of transcriptional and/or posttranslational regulation of key enzymes is challenging with regard to the recent breakthrough in deciphering oxygen sensing and signaling through NERP for protein degradation and the involvement of ERF transcription factors of the group VII as major players in the organization of the metabolic response. Based on the results of Baena-González et al. (2007) and Hinz et al. (2010), it is tempting to hypothesize that AlaAT is regulated by two convergent signaling pathways (Fig. 2). Consistently being under the control of two convergent signaling pathways may explain why AlaAT, that is under the control of RAP2.2, a substrate of NERP, was not constitutively expressed in Arabidopsis knockout mutants ate 1 , ate 2 and $p r 6$, unlike anaerobic marker genes $A D H 1, P D C 1$ and SUS1/4. The idea that other mechanisms through or together with NERP are responsible for tolerance of plants to variations in oxygen was proposed and suggested to be the most challenging aspect in this field (Licausi et al. 2013). Therefore, dissecting hypoxia and darkness signaling pathways in Arabidopsis shoots seems an opportunity for deciphering how NERP through RAP2.2 interacts with another signaling pathway, namely darkness through KIN10/KIN11, for regulating one of the aspects of metabolic response to oxygen deprivation (Fig. 2).

Finally, the hypothesis of GDH posttranslational regulation by phosphorylation deserves to be challenged; it is very likely that the activity of this enzyme needs a finetune regulation for the rapid switch from inhibited to active state during transition from low oxygen stress to post-stress recovery period. Our hypothesis is that carbon stored in the form of alanine during low oxygen stress is mobilized through an AlaAT/GDH cycle; pyruvate and glutamate are generated by AlaAT from alanine and 2-oxoglutarate, pyruvate is funneled to the Krebs cycle, while the oxidative deamination of glutamate by GDH restores 2-oxoglutarate 
for maintaining the cycle and generates NADH which, on oxidation, produces energy.

Acknowledgments This article is dedicated to Claudie Ricoult in memoriam. The authors wish to thank Dr Bertrand Hirel for critical reading of the manuscript and suggestions of improvement. Many thanks also to Pr Peter Neauman for critical reading the manuscript and English language correction. Our work on root hypoxia was funded by QUALISEM program funded by 'Region Pays de la Loire', France.

\section{References}

Albrecht G, Mustroph A, Theodore C (2004) Sugar and fructan accumulation during metabolic adjustment between respiration and fermentation under low oxygen conditions in wheat roots. Physiol Plant 120:93-104

Allègre A, Silvestre J, Morard P, Kallerhoff J, Pinelli E (2004) Nitrate reductase regulation in tomato roots by exogenous nitrate: a possible role in tolerance to long-term root anoxia. J Exp Bot 55:2625-2634

Baena-González E, Rolland F, Thevelein JM, Sheen J (2007) A central integrator of transcription networks in plant stress and energy signalling. Nature 448:938-942

Bailey-Serres J, Voesenek LA (2008) Flooding stress: acclimations and genetic diversity. Annu Rev Plant Biol 59:313-339

Bailey-Serres J, Fukao T, Gibbs DJ, Holdsworth MJ, Lee SC, Licausi F, Perata P, Voesenek LA, van Dongen JT (2012) Making sense of low oxygen sensing. Trends Plant Sci 17:129-138

Benamar A, Rolletschek H, Borisjuk L, Avelange-Macherel MH, Curien G, Mostefai HA, Andriantsitohaina R, Macherel D (2008) Nitrite-nitric oxide control of mitochondrial respiration at the frontier of anoxia. Biochim Biophys Acta 1777:1268-1275

Besson-Bard A, Courtois C, Gauthier A, Dahan J, Dobrowolska G, Jeandroz S, Pugin A, Wendehenne D (2008a) Nitric oxide in plants: production and cross-talk with $\mathrm{Ca} 2+$ signaling. Mol Plant 1:218-228

Besson-Bard A, Pugin A, Wendehenne D (2008b) New insights into nitric oxide signaling in plants. Annu Rev Plant Biol 59:21-39

Botrel A, Kaiser WM (1997) Nitrate reductase activation state in barley roots in relation to the energy and carbohydrate status. Planta 201:496-501

Bouché N, Fait A, Zik M, Fromm H (2004) The root-specific glutamate decarboxylase (GAD1) is essential for sustaining GABA levels in Arabidopsis. Plant Mol Biol 55:315-325

Bouché N, Yellin A, Snedden WA, Fromm H (2005) Plant-specific calmodulin-binding proteins. Annu Rev Plant Biol 56:435-466

Bright J, Desikan R, Hancock JT, Weir IS, Neill SJ (2006) ABAinduced NO generation and stomatal closure in Arabidopsis are dependent on $\mathrm{H} 2 \mathrm{O} 2$ synthesis. Plant J 45:113-122

Buwalda W, Greeway H (1989) Nitrogen uptake and growth of wheat during $\mathrm{O} 2$ deficiency in root media containing $\mathrm{NO}_{3}{ }^{-}$only, or $\mathrm{NO}_{3}^{-}$plus $\mathrm{NH}_{4}^{+}$. New Phytol 111:161-166

Carroll AD, Fox GG, Laurie S, Phillips R, Ratcliffe RG, Stewart GR (1994) Ammonium assimilation and the role of [gamma]-aminobutyric acid in $\mathrm{pH}$ homeostasis in carrot cell suspensions. Plant Physiol 106:513-520

Dawson NJ, Storey KB (2012) An enzymatic bridge between carbohydrate and amino acid metabolism: regulation of glutamate dehydrogenase by reversible phosphorylation in a severe hypoxia-tolerant crayfish. J Comp Physiol B 182:331-340

Dordas C, Hasinoff BB, Igamberdiev AU, Manac'h N, Rivoal J, Hill RD (2003a) Expression of a stress-induced hemoglobin affects
NO levels produced by alfalfa root cultures under hypoxic stress. Plant J 35:763-770

Dordas C, Rivoal J, Hill RD (2003b) Plant haemoglobins, nitric oxide and hypoxic stress. Ann Bot 91:173-178

Drew MC (1997) Oxygen deficiency and root metabolism: injury and acclimation under hypoxia and anoxia. Ann Rev Plant Physiol Plant Mol Biol 48:223-250

Felle HH (2006) Apoplastic pH during low-oxygen stress in Barley. Ann Bot 98:1085-1093

Garcia-Mata C, Gay R, Sokolovski S, Hills A, Lamattina L, Blatt MR (2003) Nitric oxide regulates $\mathrm{K}+$ and $\mathrm{Cl}-$ channels in guard cells through a subset of abscisic acid-evoked signaling pathways. Proc Natl Acad Sci USA 100:11116-11121

Gibbs DJ, Lee SC, Isa NM, Gramuglia S, Fukao T, Bassel GW, Correia CS, Corbineau F, Theodoulou FL, Bailey-Serres J, Holdsworth MJ (2011) Homeostatic response to hypoxia is regulated by the N-end rule pathway in plants. Nature 479:415-418

Gout E, Boisson A, Aubert S, Douce R, Bligny R (2001) Origin of the cytoplasmic $\mathrm{pH}$ changes during anaerobic stress in higher plant cells. Carbon-13 and phosphorous-31 nuclear magnetic resonance studies. Plant Physiol 125:912-925

Greenway H, Gibbs J (2003) Mechanisms of anoxia tolerance in plants. II. Energy requirements for maintenance and energy distribution to essential processes. Funct Plant Biol 30:999-1036

Gupta KJ, Igamberdiev AU (2011) The anoxic plant mitochondrion as a nitrite: NO reductase. Mitochondrion 11:537-543

Hattori Y, Nagai K, Furukawa S, Song XJ, Kawano R, Sakakibara H, Wu J, Matsumoto T, Yoshimura A, Kitano H, Matsuoka M, Mori H, Ashikari M (2009) The ethylene response factors SNORKEL1 and SNORKEL2 allow rice to adapt to deep water. Nature 460:1026-1030

Hebelstrup KH, van Zanten M, Mandon J, Voesenek LA, Harren FJ, Cristescu SM, Møller IM, Mur LA (2012) Haemoglobin modulates $\mathrm{NO}$ emission and hyponasty under hypoxia-related stress in Arabidopsis thaliana. J Exp Bot 63:5581-5591

Hess N, Klode M, Anders M, Sauter M (2011) The hypoxia responsive transcription factor genes ERF71/HRE2 and ERF73/HRE1 of Arabidopsis are differentially regulated by ethylene. Physiol Plant 143:41-49

Hinz M, Wilson IW, Yang J, Buerstenbinder K, Llewellyn D, Dennis ES, Sauter M, Dolferus R (2010) Arabidopsis RAP2.2: an ethylene response transcription factor that is important for hypoxia survival. Plant Physiol 153:757-772

Horchani F, Aschi-Smiti S, Brouquisse R (2010) Involvement of nitrate reduction in the tolerance of tomato (Solanum lycopersicum L.) plants to prolonged root hypoxia. Acta Physiol Plant 32:1113-1123

Horchani F, Prévot M, Boscari A, Evangelisti E, Meilhoc E, Bruand C, Raymond P, Boncompagni E, Aschi-Smiti S, Puppo A, Brouquisse R (2011) Both plant and bacterial nitrate reductases contribute to nitric oxide production in Medicago truncatula nitrogen-fixing nodules. Plant Physiol 155:1023-1036

Hu RG, Sheng J, Qi X, Xu Z, Takahashi TT, Varshavsky A (2005) The $\mathrm{N}$-end rule pathway as a nitric oxide sensor controlling the levels of multiple regulators. Nature 437:981-986

Igamberdiev AU, Hill RD (2004) Nitrate, NO and haemoglobin in plant adaptation to hypoxia: an alternative to classic fermentation pathways. J Exp Bot 55:2473-2482

Igamberdiev AU, Hill RD (2009) Plant mitochondrial function during anaerobiosis. Ann Bot 103:259-268

Igamberdiev AU, Seregélyes C, Manac'h N, Hill RD (2004) NADHdependent metabolism of nitric oxide in alfalfa root cultures expressing barley hemoglobin. Planta 219(1):95-102

Igamberdiev AU, Bykova NV, Shah JK, Hill RD (2010) Anoxic nitric oxide cycling in plants: participating reactions and possible mechanisms. Physiol Plant 138:393-404 
Ismond KP, Dolferus R, De Pauw M, Dennis ES, Good AG (2003) Enhanced low oxygen survival in Arabidopsis through increased metabolic flux in the fermentative pathway. Plant Physiol 132:1292-1302

Jackson M (1985) Ethylene and responses of plants to soil waterlogging and submergence. Ann Rev Plant Physiol Plant Mol Biol $36: 145-174$

Jackson M, Armstrong W (1999) Formation of aerenchyma and the processes of plant ventilation in relation to soil flooding and submergence. Plant Biol 1:274-287

Jacoby RP, Li L, Huang S, Pong Lee C, Millar AH, Taylor NL (2012) Mitochondrial composition, function and stress response in plants. J Integr Plant Biol 54:887-906

Kaiser WM, Weiner H, Kandlbinder A, Tsai CB, Rockel P, Sonoda M, Planchet E (2002) Modulation of nitrate reductase: some new insights, an unusual case and a potentially important side reaction. J Exp Bot 53:875-882

Klok EJ, Wilson IW, Wilson D, Chapman SC, Ewing RM, Somerville SC, Peacock WJ, Dolferus R, Dennis ES (2002) Expression profile analysis of the low-oxygen response in Arabidopsis root cultures. Plant Cell 14:2481-2494

Kursteiner O, Dupuis I, Kuhlemeier C (2003) The pyruvate decarboxylase1 gene of Arabidopsis is required during Anoxia but not other environmental stresses. Plant Physiol 132:968-978

Lamotte O, Gould K, Lecourieux D, Sequeira-Legrand A, LebrunGarcia A, Durner J, Pugin A, Wendehenne D (2004) Analysis of nitric oxide signaling functions in tobacco cells challenged by the elicitor cryptogein. Plant Physiol 135:516-529

Lasanthi-Kudahettige R, Magneschi L, Loreti E, Gonzali S, Licausi F, Novi G, Beretta O, Vitulli F, Alpi A, Perata P (2007) Transcript profiling of the anoxic rice coleoptile. Plant Physiol 144:218-231

Lee KW, Chen PW, Lu CA, Chen S, Ho TH, Yu SM (2009) Coordinated responses to oxygen and sugar deficiency allow rice seedlings to tolerate flooding. Sci Signal 2:ra61

Libourel IG, van Bodegom PM, Fricker MD, Ratcliffe RG (2006) Nitrite reduces cytoplasmic acidosis under anoxia. Plant Physiol 142:1710-1717

Licausi F (2013) Molecular elements of low-oxygen signaling in plants. Physiol Plant 148:1-8

Licausi F, van Dongen JT, Giuntoli B, Novi G, Santaniello A, Geigenberger P, Perata P (2010) HRE1 and HRE2, two hypoxiainducible ethylene response factors, affect anaerobic responses in Arabidopsis thaliana. Plant J 62:302-315

Licausi F, Kosmacz M, Weits DA, Giuntoli B, Giorgi FM, Voesenek LA, Perata P, van Dongen JT (2011) Oxygen sensing in plants is mediated by an $\mathrm{N}$-end rule pathway for protein destabilization. Nature 479:419-422

Licausi F, Pucciariello C, Perata P (2013) New role for an old rule: $\mathrm{N}$-end rule-mediated degradation of ethylene responsive factor proteins governs low oxygen response in plants $(\mathrm{F})$. J Integr Plant Biol 55:31-39

Limami AM, Glevarec G, Ricoult C, Cliquet J-B, Planchet E (2008) Concerted modulation of alanine and glutamate metabolism in young Medicago truncatula seedlings under hypoxic stress. J Exp Bot 59:2325-2335

Liu F, Vantoai T, Moy LP, Bock G, Linford LD, Quackenbush J (2005a) Global transcription profiling reveals comprehensive insights into hypoxic response in Arabidopsis. Plant Physiol 137:1115-1129

Liu F, VanToai T, Moy LP, Bock G, Linford LD, Quackenbush J (2005b) Global transcription profiling reveals comprehensive insights into hypoxic response in Arabidopsis. Plant Physiol 137:1115-1129. doi:10.1104/pp.104.055475

Loreti E, Poggi A, Novi G, Alpi A, Perata P (2005) A genome-wide analysis of the effects of sucrose on gene expression in Arabidopsis seedlings under anoxia. Plant Physiol 137:1130-1138
Ma F, Lu R, Liu H, Shi B, Zhang J, Tan M, Zhang A, Jiang M (2012) Nitric oxide-activated calcium/calmodulin-dependent protein kinase regulates the abscisic acid-induced antioxidant defence in maize. J Exp Bot 63:4835-4847

Melo-Oliveira R, Oliveira IC, Coruzzi GM (1996) Arabidopsis mutant analysis and gene regulation define a nonredundant role for glutamate dehydrogenase in nitrogen assimilation. Proc Natl Acad Sci USA 93:4718-4723

Morard P, Silvestre J, Lacoste L, Caumes E, Lamaze T (2004) Nitrate uptake and nitrite release by tomato roots in response to anoxia. $\mathbf{J}$ Plant Physiol 161:855-865

Mustroph A, Zanetti ME, Jang CJ, Holtan HE, Repetti PP, Galbraith DW, Girke T, Bailey-Serres J (2009) Profiling translatomes of discrete cell populations resolves altered cellular priorities during hypoxia in Arabidopsis. Proc Natl Acad Sci USA 106:18843-18848

Mustroph A, Lee SC, Oosumi T, Zanetti ME, Yang H, Ma K, Yaghoubi-Masihi A, Fukao T, Bailey-Serres J (2010) Cross-kingdom comparison of transcriptomic adjustments to low-oxygen stress highlights conserved and plant-specific responses. Plant Physiol 152:1484-1500

Narsai R, Howell KA, Carroll A, Ivanova A, Millar AH, Whelan J (2009) Defining core metabolic and transcriptomic responses to oxygen availability in rice embryos and young seedlings. Plant Physiol 151:306-322

Oliveira HC, Sodek L (2013) Effect of oxygen deficiency on nitrogen assimilation and amino acid metabolism of soybean root segments. Amino Acids 44:743-755

Oliveira HC, Freschi L, Sodek L (2013) Nitrogen metabolism and translocation in soybean plants subjected to root oxygen deficiency. Plant Physiol Biochem 66:141-149

Planchet E, Jagadis Gupta K, Sonoda M, Kaiser WM (2005) Nitric oxide emission from tobacco leaves and cell suspensions: rate limiting factors and evidence for the involvement of mitochondrial electron transport. Plant J 41:732-743

Ricoult C, Cliquet J-B, Limami AM (2005) Stimulation of alanine amino transferase (AlaAT) gene expression and alanine accumulation in embryo axis of the model legume Medicago truncatula contribute to anoxia stress tolerance. Physiol Plant 123:30-39

Ricoult C, Echeverria LO, Cliquet JB, Limami AM (2006) Characterization of alanine aminotransferase (AlaAT) multigene family and hypoxic response in young seedlings of the model legume Medicago truncatula. J Exp Bot 57:3079-3089

Rocha M, Licausi F, Araujo WL, Nunes-Nesi A, Sodek L, Fernie AR, van Dongen JT (2010) Glycolysis and the tricarboxylic acid cycle are linked by Alanine Aminotransferase during hypoxia induced by waterlogging of Lotus japonicus. Plant Physiol 152:1501-1513

Rockel P, Strube F, Rockel A, Wildt J, Kaiser WM (2002) Regulation of nitric oxide (NO) production by plant nitrate reductase in vivo and in vitro. J Exp Bot 53:103-110

Sakihama Y, Nakamura S, Yamasaki H (2002) Nitric oxide production mediated by nitrate reductase in the green alga Chlamydomonas reinhardtii: an alternative NO production pathway in photosynthetic organisms. Plant Cell Physiol 43:290-297

Sasidharan R, Mustroph A (2011) Plant oxygen sensing is mediated by the N-end rule pathway: a milestone in plant anaerobiosis. Plant Cell 23:4173-4183

Shi K, Ding X-T, Don D-K, Zhou Y-H, JQ Y (2008) Putrescine enhancement of tolerance to root-zone hypoxia in Cucumis sativus: a role in increased nitrate reductase. Funct Plant Biol 35:337-385

Shingaki-Wells RN, Huang S, Taylor NL, Carroll AJ, Zhou W, Millar AH (2011) Differential molecular responses of rice and wheat coleoptiles to anoxia reveal novel metabolic adaptations in amino acid metabolism for tissue tolerance. Plant Physiol 156:1706-1724 
Stoimenova M, Hansch R, Mendel R, Gimmler H, WM K (2003) The role of nitrate reduction in the anoxic metabolism of roots. I. Characterization of root morphology and normoxic metabolism of wild type tobacco and a transformant lacking root nitrate reductase. Plant Soil 253:145-153

Stoimenova M, Igamberdiev AU, Gupta KJ, Hill RD (2007) Nitritedriven anaerobic ATP synthesis in barley and rice root mitochondria. Planta 226:465-474

Talwar PS, Gupta R, Maurya AK, Deswal R (2012) Brassica juncea nitric oxide synthase like activity is stimulated by $\mathrm{PKC}$ activators and calcium suggesting modulation by PKC-like kinase. Plant Physiol Biochem 60:157-164

van Dongen JT, Fröhlich A, Ramírez-Aguilar SJ, Schauer N, Fernie AR, Erban A, Kopka J, Clark J, Langer A, Geigenberger P (2009) Transcript and metabolite profiling of the adaptive response to mild decreases in oxygen concentration in the roots of arabidopsis plants. Ann Bot 103:269-280
Xu K, Xu X, Fukao T, Canlas P, Maghirang-Rodriguez R, Heuer S, Ismail AM, Bailey-Serres J, Ronald PC, Mackill DJ (2006) Sub1A is an ethylene-response-factor-like gene that confers submergence tolerance to rice. Nature 442:705-708

Yamasaki H, Sakihama Y, Takahashi S (1999) An alternative pathway for nitric oxide production in plants: new features of an old enzyme. Trends Plant Sci 4:128-129

Yang CY, Hsu FC, Li JP, Wang NN, Shih MC (2011) The AP2/ERF transcription factor AtERF73/HRE1 modulates ethylene responses during hypoxia in Arabidopsis. Plant Physiol 156:202-212

Zhang A, Jiang M, Zhang J, Ding H, Xu S, Hu X, Tan M (2007) Nitric oxide induced by hydrogen peroxide mediates abscisic acid-induced activation of the mitogen-activated protein kinase cascade involved in antioxidant defense in maize leaves. New Phytol 175:36-50 